\title{
La experiencia del asombro: una aproximación expansiva a la estética cotidiana*
}

\begin{abstract}
Resumen
A diferencia de Melchionne (2013) y Naukkarinen (2013), abogo por una definición expansiva de la estética cotidiana que, además de las experiencias más comunes y cotidianas, incluya los festivales, el turismo y un sinnúmero de actividades llevadas a cabo diariamente por artistas y profesionales de diversas áreas. Establezco continuidades entre las estéticas de la vida diaria, del arte y de la naturaleza. Por ejemplo, el acto de mirar a través de una ventana puede involucrar aspectos de las tres estéticas. Aunque coincido con Melchionne (2013) en que la estética cotidiana está muy ligada a las preguntas por la búsqueda del bienestar subjetivo, asumo una aproximación más expansiva basada en estudios sicológicos recientes sobre la experiencia del asombro; ello con el fin de enfatizar la importancia de esa experiencia en tal búsqueda, vinculando así, los aspectos más sobresalientes de la estética cotidiana, la estética del arte y la estética de la naturaleza.
\end{abstract}

\footnotetext{
* Esta traducción se inscribe en la investigación Estética cotidiana y literatura, Ilevada a cabo con el apoyo del Centro de Investigación en Comunicación, CIC, de la Universidad de Medellín.

Artículo publicado originalmente en Contemporary Aesthetics, volumen 13, octubre 6, 2015. https://digitalcommons.risd.edu/liberalarts_contempaesthetics/vol13/iss1/8/
}

Thomas Leddy

Ph.D. en filosofía

San José State University, USA

Correo electrónico:

thomas.leddy@sjsu.edu

Google Scholar

Horacio Pérez-Henao

Ph.D. en Educación

Traductor (Universidad de Medellín)

Medellín, Colombia

Correo electrónico:

hperez@udem.edu.co

๑ orcid.org/0000-0001-6521-4382

Google Scholar

Recibido: julio 01 de 2018

Aprobado: abril 14 de 2020

\section{Palabras clave:}

aura, definición, estética

cotidiana, Melchionne,

Naukkarinen, restrictivos, bienestar subjetivo. 


\section{The experience of awe: an expansive approach to everyday aesthetics}

\begin{abstract}
Unlike Melchionne (2013) and Naukkarinen (2013), I advocate an expansive definition of everyday aesthetics that, in addition to the most common and everyday experiences, includes festivals, tourism, and countless activities carried out daily by artists and professionals from various areas. Continuities between aesthetics of everyday life and the aesthetics of art and nature are established. For example, the act of looking through a window may involve aspects of all three aesthetics. Although I agree with Melchionne (2013) in that everyday aesthetics is closely linked to questions about the search for subjective well-being, I assume a more expansive approach based on recent psychological studies of the experience of "awe" to emphasize the importance of such experiences in subjective search, linking this way, the most outstanding aspects of everyday aesthetics, the aesthetics of art and the aesthetics of nature.
\end{abstract}

Key words:

awe, definition, everyday aesthetics, Melchionne, Naukkarinen, restrictive, subjective well-being. 


\section{Antecedentes del debate sobre la definición de estética cotidiana}

Cuando titulé mi libro Lo extraordinario en lo ordinario (Leddy, 2012a), intenté contrarrestar una tendencia en la estética cotidiana que limita esta nueva y creciente disciplina al dominio de lo común y lo ordinario'. Pensaba que esa limitación no lograba captar la interacción dinámica entre la estética cotidiana, la estética de la naturaleza y la estética del arte. Mientras otros buscaban subrayar las discontinuidades entre tales disciplinas, yo quería resaltar las continuidades entre ellas. Siguiendo los postulados de Dewey (2005), encontraba una relación muy estrecha entre las experiencias estéticas derivadas de las artes y las que surgían de la vida diaria. El arte reúne e intensifica las cualidades estéticas presentes en los asuntos no artísticos de la vida. Además, como lo anunció Wilde (s.f), la vida a menudo imita el arte. Frecuentemente, los objetos y actividades cotidianos pueden transcender su carácter ordinario, incluso sin la mediación del arte. Digamos, entonces, que bien sea por la intervención o no del arte, esos objetos y actividades ordinarios pueden ser vistos de una manera que les dé mayor significación, haciéndolos, algunas veces sorprendentemente, objetos del asombro, o al menos de fascinación.

A menudo, esto sucede al percibir un objeto tal como lo vería un artista, pero en ocasiones acontece de manera espontánea. Tomemos el ejemplo de la belleza humana. En nuestra vida hay momentos en los que vislumbramos a una persona que tiene una gran belleza². Claro, esto no pasa a diario, sin embargo, ¿cómo se catalogaría? La experiencia no encaja muy bien en la dicotomía tradicional de estética del arte versus estética de la naturaleza. Cuando alguien encuentra a una persona de "gran belleza", ello no es una cuestión de apreciación de la naturaleza más de lo que es de apreciación del arte. La estética de la naturaleza tiene que ver con entornos naturales; la gran belleza humana no, o, al menos,

${ }^{1}$ Un ejemplo de la postura que enfatiza lo ordinario es el trabajo de Haapala (2005). También recomiendo ver Saito (2010).

${ }^{2}$ Ver, por ejemplo, el relato Sueños de invierno de Fitzgerald (2011), donde uno de los personajes se refiere a otro como "una gran belleza". 
no directamente. Hay otros asuntos que nos suceden en contextos no artísticos ni naturales, y que son relevantes en la experiencia estética; por ejemplo, la forma en que un olor puede evocar un mundo. Pienso que esa relevancia debería ayudar a definir el campo de la estética cotidiana ${ }^{3}$.

Inspirado por Walter Benjamin, pero sin seguir sus postulados, denominé "aura" a la ya mencionada cualidad que eleva la significación de los objetos. Definí la estética en general, y la experiencia estética en particular, en términos de aura. Con la expresión mayor significación no me refería a dar mayor sentido de importancia, sino a algo más cercano a "impresionante", en su forma más intensa; "fascinante", en un nivel más débil, e "interesante," en un nivel aún más débil. Algo tiene aura o una mayor significación si parece más vivo, más real, más presente, o más conectado con otras cosas. Argumenté, para entonces, que cuando un objeto, evento o experiencia poseen una cualidad estética, por ejemplo, belleza, gracia o elegancia, es porque tiene aura.

Sin embargo, no estaba interesado solamente en momentos estéticos especiales, recubiertos por esas cualidades. También me Ilamaba la atención la omnipresencia de la experiencia estética. En otras palabras, no solo me atraía la forma en la cual incluso la cosa más ordinaria podría convertirse en extraordinaria, sino también, en experiencias estéticas inferiores, como el placer que obtenemos al contemplar un jardín bien cuidado. Estas experiencias hacen parte de la estética cotidiana al igual que los tipos de experiencias más raras e intensas que Dewey (2005) Ilamó "una experiencia". En un artículo posterior a mi libro, tales experiencias existen en un continuum en el cual las experiencias inferiores también tienen aura, aunque es menos intensa (Leddy,

\footnotetext{
${ }^{3}$ Moore (1996) formula, de manera interesante, algunos puntos paralelos desde la perspectiva de un sicólogo humanista cuando dice: "A la larga, lo que más satisface el alma es aquello cautivante, fascinante y encantador" (189). El mismo título de su libro, El reencantamiento de la vida cotidiana, de donde sale el fragmento anterior, hace un llamado a re-encantar la vida diaria, en la cual encontremos más cosas satisfactorias a través de una percepción del mundo más erótica, mágica, encantadora, mitológica y ritualista. A diferencia de quienes pondrían el ritual por fuera de la estética cotidiana, Moore desea traerlo de regreso.
} 
2012b). Mi definición de estética cotidiana era expansiva en tanto que incluía el rango entero de este continuum. Por ejemplo, con base en Lintott (2012), me atrevería a incluir en la estética cotidiana, la sublime experiencia de dar a luz, a pesar de que es algo que no pasa todos los días ni le acontece a todo el mundo. La importancia de incluir experiencias sublimes en la estética cotidiana la argumenté en mi libro (Leddy, 2012a, pp. 237-258) ${ }^{4}$

\section{En busca de una definición}

Algunos teóricos han sustentado que el campo de la estética cotidiana es demasiado amplio, y por ello ameritaría una definición más precisa. En la revista académica digital Contemporary Aesthetics, Melchionne (2013) y Naukkarinen (2013) han propuesto, cada uno por su lado, una definición restrictiva; en particular, limitando la estética cotidiana a lo ordinario, común y, casi estrictamente hablando, a lo que acontece diariamente. Como ya lo sugerí, mi postura toma un rumbo opuesto, a que podríamos llamar "expansionista". ¿Es solo cuestión de semántica o de una elección arbitraria de los límites conceptuales? No lo creo. Existen razones profundas para discrepar, y en lo que sigue trataré de dar algunas claridades. Mi discusión aquí estará dirigida, esencialmente, a los postulados de Melchionne (2013, 2014).

La definición restrictiva que Melchionne (2013) da sobre la estética cotidiana es, en parte, una reacción a la definición mía (Leddy, 2012a). Según Melchionne (2013), yo asumo la estética cotidiana como una especie de "bolsa alterna" en la cual pongo lo que no puede categorizarse como bellas artes o belleza natural. Esto no es totalmente cierto, pues también incluí algunos elementos propios de la estética cotidiana, ya que parte de mi aproximación era demostrativa. Sin embargo, mi definición atendió a un argumento de negación. Dije que la estética cotidiana cubría aquello que estaba por fuera de los dominios de 
las estéticas del arte y la naturaleza. Ahora pienso que la segunda parte del carácter negativo de mi enfoque era demasiado amplia. En primer lugar, no es tan claro decir que los fenómenos estéticos por fuera del arte y la naturaleza deberían incluirse en la estética cotidiana. Por ejemplo, ¿qué podemos decir de la estética de las matemáticas? ¿Hace parte de la estética cotidiana debido a que no pertenece a las estéticas del arte o la naturaleza? Otros campos que plantean problemas similares tienen que ver con la estética del deporte y la estética de la ciencia. En segundo lugar, una definición debería llegar a la esencia del objeto de estudio o, al menos, al significado central del concepto. Definiciones basadas en la negación no contribuyen en ninguna de las dos direcciones anteriores. Y tercero, como lo veremos más adelante, la relación de la estética cotidiana con las estéticas del arte y la naturaleza es demasiado dinámica para ser consignada en una definición negativa.

No obstante, para Melchionne (2013) el problema no es que mi definición sea negativa sino expansiva. En particular, le preocupa que este enfoque incluya asuntos que no son comunes a la mayoría de la gente, ni tienen el carácter de la repetición diaria. Esto es lo que se entiende por preferir una definición restrictiva de la estética cotidiana. Como ha de advertirse, abogaré por una aproximación expansiva para entender la estética cotidiana, toda vez que no elaboraré una definición como tal. A pesar de que las definiciones filosóficas son valiosas, el proceso para crearlas, en la medida en que implica formular distinciones rigurosas, tiende a ocultar continuidades e interacciones dinámicas, cuya comprensión, en ocasiones, es más importante que el establecimiento de límites.

Algunos podrían sugerir que el desacuerdo es fácil de resolver. Bastaría con que yo cediera el término "estética cotidiana" a quienes reclaman una definición restrictiva, y acuñara otro concepto, digamos "estética del festejo"5, 
que aglutine eventos no cotidianos, incluidos en mi enfoque de la estética cotidiana, pero rechazados por los defensores de la definición restrictiva. Por su lado, Naukkarinen (2013), quien coincide con algunas ideas de Melchionne (2013), postula una estética cotidiana centrada en lo familiar, fácil u obvio, y en las rutinas diarias hechas casi que automáticamente. Además, afirma que para él y otras personas, "los objetos, actividades y eventos cotidianos son aquellos en los que, regular y repetidamente, invertimos mucho tiempo. Muy a menudo, esto significa objetos y eventos relacionados con nuestros trabajo, casa y pasatiempos" (Naukkarinen, 2013, p. 2). Naukkarinen (2013), como Melchionne (2013), saca las fiestas de lo cotidiano porque rompen con las rutinas de la vida: "Ellas son excepciones, ocasiones en las que hacemos cosas distintas a las normales" (3). En una gráfica que acompaña la explicación de sus ideas, Naukkarinen (2013) instala la palabra "fiesta" en un círculo exterior, mientras que el concepto de "estética cotidiana" lo relaciona con lo que él llama "mi cotidianidad ahora", y que ubica en el centro del círculo interno (1).

"Estética del festejo" tiene un uso legítimo en la medida en que puede referirse a festivales, fiestas, bodas y días feriados. No obstante, dicho término, al igual que el concepto de estética de lo común y ordinario, no sería suficiente para cubrir un campo más amplio al que quiero aludir con "estética cotidiana". Por ejemplo, "estética del festejo" no incluiría la estética del embarazo y del parto. Tampoco tendría en cuenta el deleite que experimenté esta mañana al señalarle a mi esposa una serie de hermosos dibujos hechos a tiza por un niño, o quizás por un artista, y que aparecía a intervalos de una cuadra cerca de nuestra casa. Puesto que no es claramente arte, esos dibujos podrían entran en el dominio de la estética cotidiana. En algún momento consideré emplear la categoría "estética de la vida" en remplazo de "estética cotidiana", pero desistí de ello, debido a que la vida incluye, también, la experiencia del arte y la naturaleza. Recurrir al término de "estética popular" también sería problemático, toda vez que ello vincularía el arte popular, el cual parece 
pertenecer más a la estética del arte que a la estética cotidiana. Así pues, sigo prefiriendo la denominación de "estética cotidiana" para designar una esfera más amplia. La "estética de lo común y lo ordinario" puede atender a lo que los teóricos del enfoque restrictivo quieren Ilamar "estética cotidiana".

Como lo he sugerido, los debates de este tipo son más que un asunto de "mera semántica" u organización eficiente de un campo del conocimiento. Ellos son aspectos o, quizás aún, indicadores de mayores discusiones. Creo que esto es propio de cualquier debate filosófico en general. Conceptos como "arte", "belleza" y "bueno", sobre los que razonan los filósofos, no son de orden natural ni matemático. No son definibles al estilo de "agua" o "triángulo", ni sus límites se establecen con base en el uso popular generalizado. Lo que encierran estos debates son visiones que compiten sobre asuntos más importantes. En el caso de la estética cotidiana, se trata de analizar la naturaleza de la estética en sí misma, y, como lo veremos más adelante, la naturaleza de lo que entendemos por cotidianidad. Incluso, los análisis de este tipo refieren, indirectamente, problemáticas globales, como cuál es la esencia del conocimiento, el ser y la realidad. De ahí su importancia para los estudiosos. Por ejemplo, la argumentación de la naturaleza de la estética cotidiana puede ser, en cierta medida, una inquietud por saber en qué consiste la buena vida ${ }^{6}$. De ahí que estas discusiones puedan ser descritas en términos de rivalidades entre ideologías o visiones del mundo. Imaginemos que un marxista y una feminista dan definiciones distintas de estética cotidiana. Ello indica que existen discrepancias mayores entre marxistas y feministas. Claro está que, en el largo plazo, dar con tales discrepancias resulta complicado, y, en este orden de ideas, no queda claro si mis más profundos desacuerdos sean iguales en los diversos enfoques restrictivos sobre la estética cotidiana. En el caso de Naukkarinen (2013), al menos, el principal desencuentro

${ }^{6}$ Varios investigadores actuales de la estética cotidiana han incursionado en estos terrenos más amplios. Por ejemplo, Melchionne (2014) sostiene que la mejora del bienestar subjetivo es el centro de la estética cotidiana. Igualmente, yo he enfatizado la importancia de la felicidad (Leddy, 2014). 
parece ser su mirada heideggeriana opuesta a mi visión del mundo basada en Dewey (2005).

\section{Razones del enfoque restrictivo}

Los planteamientos de quienes se instalan en la aproximación restrictiva esquivan una ampliación de la estética cotidiana a lo que no es común ni hace parte del quehacer de la vida diaria. Por ejemplo, Melchionne (2013) insiste en que la estética cotidiana no debería incluir la decoración de interiores porque esta, a diferencia del cuidado y limpieza de la casa, no es una actividad cotidiana. Ahora bien, podríamos observar este asunto de manera tal que la decoración de interiores se asuma como un "arte menor", y en tal sentido pertenecería a la estética del arte, siempre y cuando no estemos hablando de las "bellas artes". No obstante, esto ignoraría la dinámica natural en la interacción entre actividades estrictamente cotidianas, como organizar una habitación, y aquellas labores poco comunes que surgen al contratar un decorador de interiores. Melchionne (2013) es consciente de esta interacción, pero prefiere mantenerla diferencia. Y aunque no se haga profesionalmente, mucho de lo que incluyen las colecciones de afición y el arreglo y decoración de una casa es suficientemente cercano al diseño de interiores. Es más, estas labores hacen parte de la muy cotidiana tarea de poner en orden lugares u objetos; lo cual permite clarificar, principalmente, los límites establecidos entre estos actos de creación tempranos.

\section{La definición de Melchionne}

Melchionne (2013) define la estética cotidiana basado en cuatro categorías, con las cuales titula cada sección de su artículo. Estas son: "continuo", "común", "actividad" y "estético, aunque no necesariamente característico de esta cualidad". Es decir, para que algo haga parte de la estética cotidiana 
debe ser continuo, común entre la mayoría de la gente, y estar inscrito en una práctica que puede no ser estética (para Melchionne, las prácticas son fundamentales, los objetos, secundarios). Esta definición puede ser vista en términos de circunstancias necesarias y suficientes, en la cual cada condición es necesaria, y su coexistencia adecuada. Resulta fácil aceptar que las tres primeras categorías sean condiciones necesarias, pero es más difícil entender cómo la cuarta podría serlo. Esto es algo que no discutiremos aquí.

La condición de "actividad" es central en la definición de Melchionne (2013), pues es indicación de lo que hemos referido como cotidiano y común. Al enfatizar la idea de actividad, sobre todo aquella que implica una práctica, Melchionne (2013) busca darles prioridad a las prácticas sobre los objetos. Sin embargo, en su argumentación parece asignarles menos importancia a las experiencias. Esta restricción es problemática, dado que existen muchas experiencias cotidianas que no están claramente vinculadas a una práctica (por ejemplo, lo chistoso de ver una especie de vehículo cuyas partes laterales están hechas con las estacas recicladas de una cerca, algo que encuentro en mi recorrido diario). Por mi parte, siguiendo a Dewey (2005), yo doy primacía a la experiencia, tanto sobre las prácticas como sobre los objetos. Los objetos se entienden mejor como aspectos de la experiencia o elementos presentes en ella. Después de todo, solo podemos analizar prácticas y objetos en tanto hacen parte de la experiencia.

Miremos ahora la condición de lo "continuo". Para Melchionne (2013), "continuo" significa algo que se da diario o casi diariamente. Pero, ¿por qué excluir ciertos eventos que no son cotidianos, por ejemplo, actividades que son propias a los fines de semana más que a los días laborales? El hecho de que los días de trabajo sean más numerosos que los fines de semana no los privilegia para enmarcarse en la estética cotidiana. Por ejemplo, ¿por qué el paseo de los domingos ha de estar por fuera de la estética cotidiana? Ahora bien, quienes 
defienden el enfoque restrictivo admitirían el paseo de los domingos alegando que, a pesar de no ser diaria, es una actividad que se hace continuamente. Sin embargo, es la experiencia la que es estética, no la práctica. Además, algo puede ser eventual, no diario, y aún formar parte de la cotidianidad, por la simple razón de que lo inusual también acontece, y todos vemos, oímos y olemos cosas extrañas todos los días.

Y ¿qué decir de los sucesos que pasan todos los días, o al menos frecuentemente, pero sólo en ciertos contextos y épocas del año? Por ejemplo, durante la Navidad contemplo con frecuencia las luces del árbol navideño. Considero que esta experiencia entra en el campo de la estética cotidiana. Sin embargo, Melchionne (2013) insiste en que las vacaciones o días festivos, y en consecuencias la decoración y las prácticas y experiencias derivadas de esos días, están por fuera de la estética cotidiana; pues las vacaciones son eventos temporales, no cotidianos. No obstante, ya que todo evento ocurre durante una época, y muchos están marcados por el carácter específico de la misma, dejarlos por fuera de lo cotidiano parecería ser muy rígido.

Del mismo modo, el enfoque restrictivo excluiría aquello que vemos cuando viajamos como turistas. ¿Acaso no hay una cotidianidad para los turistas? Y se excluye lo especial porque no sucede a diario, ¿qué podría decirse de lo que diariamente sucede, pero tiene una cualidad especial? Qué pasa, entonces, con la casi diaria experiencia que tengo de ver conejos en mi jardín cuando salgo a recoger el periódico, algo que ocurre debido a que un vecino tiene conejos que se escapan constantemente. De algún modo, es mágico salir en la mañana y encontrarse con conejos en medio de un ambiente netamente urbano. Yo incluiría esta experiencia en la estética cotidiana, si bien es cierto no es cotidiana, ni común, ni hace parte de una práctica. 
La discrepancia puede ser sobre qué es lo que cuenta como general para una teoría. Mis contradictores podrán decir que estoy recurriendo a ejemplos raros. Sin embargo, son ejemplos de asuntos que producen experiencias de asombro y fascinación, o al menos son interesantes. Como lo he sustentado, este tipo de experiencias son tan esenciales para la estética cotidiana como para la estética de la naturaleza y la estética del arte.

La pregunta que debemos hacernos es si la estética cotidiana debe estar ligada a las costumbres. Es decir, ¿puede incluirse eventos que tienen un carácter de serendipia? Si lo cotidiano tiene que ver necesariamente con las prácticas, como Melchionne (2013) parece sugerir, ello dejaría por fuera, por ejemplo, notar la manera asombrosa como los árboles se ven en la calle durante una caminada al atardecer. Esto puede pasar todos los días, al menos para quienes les gusta salir de paseo cuando cae el sol y, ello, sin embargo, no hace parte de una práctica. Está el hábito de caminar, pero eso es algo diferente. Obvio, uno puede hablar de la práctica de mirar estéticamente, pero, justamente, esta es la práctica que permitiría advertir asuntos que son visualmente extraños o extraños a otros sentidos. Por lo tanto, si esta práctica fuera permitida, entonces apelar a los hábitos o costumbres no contribuiría con el enfoque restrictivo.

\section{Problemas con la condición de lo común}

Los problemas más relevantes surgen con la segunda condición, la de lo común. Tengamos en cuenta, y esto es algo de lo que Melchionne (2013) y Naukkarinen (2013) son conscientes: lo que es típicamente cotidiano para una persona será muy diferente para otra. La vida diaria de un monje trapense ha de ser diferente de la de alguien en otro tipo de cultura. Una manera de resolver esto es enfatizar lo que los humanos comparten en común. Melchionne (2013) realza, por ejemplo, el hecho de que todos tenemos que dormir, vestirnos (o al menos estar presentables físicamente para los demás), cocinar, consumir 
alimentos y salir a la calle. La lista es de gran ayuda, si bien deja por fuera muchas cosas que yo consideraría parte de la estética cotidiana. Por ejemplo, muchos jóvenes después de la pubertad tienen que afrontar el impulso sexual, y casi toda la gente adulta tiene que trabajar diariamente. Al igual que el dormir y el comer, nuestra vida sexual y laboral deberían tenerse en cuenta en la estética cotidiana ${ }^{8}$. Por otra parte, no es necesario limitar la estética cotidiana a la pequeña lista de prácticas que la humanidad comparte, y excluir la vasta gama de hábitos menos generalizados.

Al intentar explicar por qué la vida laboral debe excluirse, Melchionne (2013) sostiene que "muy pocos de nosotros somos pianistas. Por ello, la tarea diaria del pianista de ejercitar los dedos no es relevante para la estética cotidiana" $(2 b)^{9}$. Sin embargo, el número o porcentaje de personas vinculadas a una práctica no dice nada sobre si una actividad cuenta o no como parte de la vida diaria. Tocar piano es una parte importante de la cotidianidad de los pianistas. ¿Por qué descartar estas actividades de la vida diaria, a lo menos en lo pertinente a los pianistas?

Podría discutirse que los ejercicios con el piano hacen parte de la estética de la música y, por consiguiente, no entrarían en la estética cotidiana. En respuesta, pensemos en lo que constituye la vida diaria de un artista típico. En la medida en que estoy más familiarizado con las artes visuales, tomaré como ejemplo la vida de un pintor en su taller. ¿Es irrelevante la actividad diaria del artista a la estética cotidiana? Supongamos que en su taller no hay obras finalizadas. Al asumir esto necesitamos, al menos, una definición provisional de arte. La definición inicial que hace Dickie (1971) sobre arte, al ser muy conocida y breve, es de gran ayuda para nuestro propósito: "En sentido clasificatorio, una obra de arte es 1) un artefacto 2) sobre el cual una o varias personas,

\footnotetext{
${ }^{7}$ Cabe señalar que Naukkarinen (2013), a diferencia de Melchionne (2013), incluye la vida laboral en la estética cotidiana.

${ }^{8}$ Sobre las maneras en las que el comportamiento sexual puede tener una dimensión estética ver Berleant (1964) y Shusterman (2007).

${ }^{9}$ Naukkarinen (2013) parece más dispuesto a incluir este tipo de actividades en la estética cotidiana.
} 
actuando en nombre de cierta institución social (el mundo del arte), le han conferido el estatus de candidato para ser valorado" (101). En su definición no está la presencia del arte, pues el artista, como representante del mundo del arte, no ha señalado algo como candidato de valoración, es decir, aún no ha considerado nada disponible para ser visto por otros.

Incluso si tomamos esta definición de arte, en el taller suceden muchas cosas que tienen que ver con la estética. Pensemos en que el artista está pintando un bodegón basado en un arreglo de frutas. Al observar las frutas, el artista atiende a las propiedades estéticas de estas, posiblemente capturando o resaltándolas en el modo en que las representa. Dichas propiedades también pudieron haber sido identificadas en la cocina, antes de agrupar las frutas para su uso en el taller. En pocas palabras, las cualidades estéticas halladas por el pintor en su taller pertenecen a la estética cotidiana.

Además, en el proceso de producción de la obra de arte, el artista atiende a las cualidades estéticas de las pinturas utilizadas, incluyendo el modo como estas interactúan en el campo perceptivo, tanto en la paleta como en la superficie de la pintura. A ello se añade que el pintor puede, al menos, estar de modo subconsciente atento a la dinámica en las relaciones entre las distintas partes de su propio cuerpo mientras pinta; por ejemplo, el movimiento de sus manos y el manejo de sus ojos. Descartar cualquier cosa de estas es un error.

Lo anterior no significa confundir la estética cotidiana con la estética del arte. La estética del arte, al menos en lo que respecta a Dickie (1971), solo se da cuando lo que el artista ha creado alcanza el estatus de arte. No estoy tan seguro de que esto sea correcto, pues, incluso, cuando en el estudio o taller no existen elementos designados como objetos artísticos, está la presencia del arte en la medida en la que hay un proceso de producción artístico. Por lo tanto, podríamos, alternativamente, estar interesados en extender la 
estética del arte al proceso mismo de creación que conduce al objeto-arte. No obstante, esto indicaría una superposición entre la estética cotidiana y la estética del arte en el mundo del trabajo del artista. Pero este tipo de traslapos es ignorado en el enfoque restrictivo que pretende definir la estética cotidiana. Tanto esta última como la estética del arte se ven perjudicadas cuando se abordan de manera aislada.

El mismo Melchionne (2013) menciona algo de la dinámica relación entre la estética del arte y la estética cotidiana cuando dice que la ceremonia japonesa del té "eleva lo cotidiano a la condición de ritual", y señala que "Si después de participar en tal ceremonia, uno retoma la preparación de la comida diaria valorando más los utensilios, los aromas y el agua utilizada, entonces el ritual del té ha aumentado la estética cotidiana en mi vida" (2b). El mismo autor capta el efecto de mejoría que se produce en nuestras vidas cuando, por ejemplo, ponemos la suficiente atención a la estética cotidiana (algo de lo que hablaré más adelante). Lo que quiero indicar aquí no es que la ceremonia del té sea un ejemplo de estética cotidiana, sino que la estrecha relación entre ritual, arte y estética cotidiana es particularmente evidente en dicha ceremonia.

Complejo es el ejemplo que Melchionne (2013) pone en relación con una ventana. Con él plantea que "la ventana de una alcoba con vista hacia un paisaje no tiene valor para la estética cotidiana, si la habitación es escasamente ocupada o la persiana siempre está cerrada" (2c). Obvio, la vista no tiene valor estético si no es mirada por alguien. Tampoco la ventana como tal tiene valor estético si nunca es usada. Pero ¿qué pasa en las pocas veces cuando la habitación es ocupada? Pensemos en una cabaña en el campo, visitada una vez al año. Para Melchionne (2013), una ventana con una vista especial es muy diferente de aquella a través de la cual vemos algunas cosas diariamente. Esto último es una práctica, mientras que ver a través de la ventana en el campo una vez al año podría enmarcarse, quizá, 
en la estética festiva. De nuevo, para este autor, el valor estético del objeto y la experiencia son secundarios en relación con la práctica. No me queda claro por qué mirar a través de una ventana diariamente es una práctica, mientras que hacerlo una vez al año no lo es.

Coincido con Melchionne (2013) en que "si la luz, la vista y la silla al lado, contribuyen al carácter estético de un momento cotidiano, entonces podríamos hablar de la ventana en términos de estética cotidiana" (2c). Pero esto es igualmente cierto si tales cosas aportan la cualidad estética a una experiencia anual. Cuando Melchionne (2013) afirma que "es el café habitual de la mañana, el reconocimiento del atardecer, o el mero hecho de abrir una persiana al despertar, lo que da el carácter de estética cotidiana a la ventana" (2c), de nuevo, estoy de acuerdo, pero existen, además, otros elementos menos comunes que también contribuyen. El valor estético proviene, además, de aquello que es propio de la ventana, por ejemplo, su diseño y, en relación con la experiencia, lo que es visto en un día en particular. Adicionalmente, cada aspecto de estas cosas, cada situación específica vinculada al café de la mañana y, también, quizá, abrir la ventana al despertar, puede adquirir un aura de mayor significación, es decir, puede ser estético.

El asunto relacionado con la ventana y su vista me lleva a pensar en otro tema vinculado con la estética de la naturaleza. Así como la relación entre la estética del arte y la estética cotidiana se entiende mejor desde su dinámica, igual sucede entre la relación de estas dos con la estética de la naturaleza. El reconocimiento de lo anterior tiene profundas implicaciones en la estética en general. Una ventana con vistas a un paisaje no es en sí naturaleza, y, sin embargo, mirar el paisaje a través de la ventana tiene que ver con apreciar la naturaleza y, desde un ángulo diferente, un aspecto de la estética cotidiana. Ello también se conecta con la estética del arte por vía del arte de la arquitectura. Alguien podría argumentar que ver un paisaje a través de 
una ventana no es apreciar la naturaleza como naturaleza, sino, más bien, observarla como si fuera una pintura ${ }^{10}$. Sin embargo, dicha observación es válida siempre y cuando no se considere la única manera de ver la naturaleza. Podría pensarse que caminar entre la naturaleza es una forma más auténtica o apropiada de apreciarla, que verla a través de una ventana. Pero ello depende de qué tan a menudo o con qué cuidado se hace dicha mirada. Mirar a través de una ventana es una de las muchas opciones que tenemos para ver la naturaleza. La estética de la naturaleza debería, entonces, ser lo suficientemente amplia para encargarse de esta forma de ver.

Pero la apreciación de la naturaleza no es el punto central aquí. Ser consciente de la presencia de la ventana, el marco y su vidrio, su estructura, su solidez, su puesto en relación con la arquitectura del lugar, entre otras cosas, pueden ser elementos esenciales en la experiencia general de una persona. Mirar un paisaje a través de una ventana, entonces, es un asunto de la valoración del arte arquitectónico como también un aspecto de la apreciación de la naturaleza ${ }^{11}$. La experiencia de la ventana, y la de la escena a través de ella, está relacionada con el arte y con la naturaleza. También está vinculada con la estética cotidiana, al punto de que los elementos de la relación arte y naturaleza están contextualizados en la actividad cotidiana de "ver a través de una ventana". En pocas palabras, lo cotidiano, lo natural y lo artístico están regularmente entrelazados de formas complejas.

Por otra parte, limitar la estética cotidiana a las prácticas diarias o casi diarias es desconocer que existen concepciones ideales de estética cotidiana que no se materializan muy a menudo, y que son necesarias en la definición de la estética cotidiana. Ejemplos de dichos ideales incluyen aquellos a los que

\footnotetext{
${ }^{10}$ Es el caso de Carlson (1979).

${ }^{11}$ Yuriko Saito me ha mencionado dos buenos ejemplos: las ventanas de Frank Lloyd Wright sin ninguna estructura, y localizadas en Taliesin, Wisconsin, USA (muchas de esas ventanas han incorporado diseños de vidirieras), y la Villa Seprada Katsura (también conocida como Palacio), en Japón, que cuenta con un marco de ventana en una forma decorativa, lo que le añade un componente de apreciación estética.
} 
Dewey (2005) se refirió como "una experiencia", y las experiencias de asombro que también son estéticas.

\section{Bienestar subjetivo y asombro}

Recientemente, Melchionne (2014) ha explicado que el propósito de la estética cotidiana es el bienestar subjetivo. Esto podría asumirse como la representación de un ideal, pero ¿cómo se obtiene el bienestar subjetivo? Melchionne (2014) cree que la mejor manera de adquirirlo es a través de la regulación hedónica. Aplaudo la idea de que el bienestar subjetivo aumenta, si se pone una mayor atención a los fenómenos de la estética cotidiana. No obstante, dudo del exclusivo énfasis puesto en la regulación del placer. Ya he mencionado la importancia del concepto de asombro. Existen evidencias sicológicas recientes que sustentan la idea de que el bienestar subjetivo crece en momentos de asombro ${ }^{12}$. Tal como los sicólogos han asegurado, las circunstancias de asombro a menudo emergen como resultado de experimentar una gran belleza o sublimidad en el arte o la naturaleza. Parece, entonces, que hay algo problemático en el reclamo de Melchionne (2014) cuando dice que "las prácticas de estética cotidiana creadas por nosotros mismos tienen muchas más posibilidades de influir en el bienestar que los encuentros ocasionales con las bellas artes o el arte popular, como ir de vez en cuando a museos o conciertos" (p. 4). Las prácticas diseñadas por nosotros mismos son solo un factor en el mejoramiento de la vida. Las experiencias de asombro pueden venir de la naturaleza, el arte o contextos cotidianos.

Propongo que abordemos la estética cotidiana, la estética del arte y la estética de la naturaleza en igual de condiciones cuando se trate de incentivar el bienestar personal. En primer lugar, nuestros encuentros con el arte popular, al menos en

\footnotetext{
${ }^{12}$ Ver Piff \& Keltner (2015). La periodista en sicología, Ana Mikulak señala que "en dos estudios, Van Cappellen y sus colegas (2014), encontraron que las emociones positivas de auto trascendencia, incluyendo el asombro, mediaban la asociación observada entre religiosidad, espiritualidad y bienestar" (Milukak, 2015).
} 
países industrializados como los Estados Unidos, no son tan ocasionales y, de hecho, toman gran parte de los días de la gente. Segundo, a pesar de que las experiencias de asombro son casuales, no son excepcionales. Es más, puede asegurarse que dichas experiencias dan en gran medida significado a la vida. Por otra parte, tales experiencias, a pesar de que son inducidas regularmente por el arte y la naturaleza, también son motivadas muy a menudo por contextos no artísticos ni naturales.

Podría contraargumentarse que hablar del asombro es tan solo otra manera de hacer referencia al peldaño superior de la escala hedónica. Pero el asombro y la relación más estrecha que guarda con el concepto de fascinación, no simplemente marcan el nivel más alto de placer. Más bien, al igual que las experiencias de lo sublime, tienen aspectos negativos o perturbadores, y otras veces positivos; aunque agradables en cierto sentido, habría que reconocer que el placer es un asunto complejo.

¿Pero es el asombro estético? Puede serlo, aunque no necesariamente. El diccionario lo define como "abrumadora maravilla, admiración, respeto o temor" $^{\prime 13}$. No parece haber un componente de placer o deleite requerido aquí en la forma en que Burke (1990) requirió "deleite" para que lo sublime fuera estético ${ }^{14}$. Sin embargo, las maravillas, la admiración y el respeto podrían tener cada uno su propio efecto positivo asociado, y, quizá, debido a que yo no practico religión alguna, no puedo imaginarme personalmente el componente positivo del temor. El diccionario.com sugiere que el sentido actual que adquiere el concepto de asombro, en la medida en que mezcla temor y veneración, se debe a su uso bíblico en referencia a Dios. La veneración puede tener un componente de afecto positivo, y cualquier temor que un creyente tenga hacia Dios puede ser combinado con algún afecto positivo, por ejemplo,

\footnotetext{
13 "Awe" Dictionary. com http://www.dictionary.com/browse/awe, visitado en junio 8 de 2015.

${ }^{14}$ Ver Burke (1990), Section IV, "Of Delight and Pleasure, as Opposed to Each Other", pp. 33-34.
} 
el amor. De lo contrario, ¿por qué adorarlo? No obstante, si el asombro fuera definido como simplemente una combinación de miedo y sorpresa, como algunos lo han hecho ${ }^{15}$, entonces solo podría relacionarse tangencialmente con la estética; al menos que el aspecto sorpresa también contenga, por supuesto, un elemento de deleite. Asimismo, los sicólogos han observado que cuando la gente describe las experiencias de asombro, tales experiencias son comúnmente consideradas positivas.

Al estilo de los análisis sobre el arte hechos por esteticistas contemporáneos como Dutton (2010), Davies (2015) y Dissanayake (1992), los sicólogos Piff y Keltner (2015) formulan un recuento evolutivo de la experiencia del asombro. Para ellos, el asombro incita a la gente a tomar parte en la construcción de comunidad a través de actividades como rituales colectivos, celebraciones, música, danza, encuentros religiosos y la oración (Piff \& Keltner 2015); y en tal sentido, el asombro puede ser adaptable a estas situaciones. Todos estos eventos tienen fuertes componentes estéticos. De ahí que el asombro, más amplio que lo sublime, sea uno de los fenómenos estéticos con el cual se pueda establecer relaciones múltiples. Algunos podrían rechazar esta mezcla entre lo estético y lo religioso, pero es una combinación que también se presenta en lo sublime. No obstante, en las sociedades tribales (de las cuales descendemos todos los que no somos tribales), las artes, la música, la danza y los rituales no están claramente diferenciados de lo religioso. De tal modo que la distinción entre lo uno y lo otro puede ser relativamente nueva y relativamente sin importancia al discutir ampliamente sobre la estética.

Los sicólogos a los que me he referido asocian el asombro con un cambio de enfoque que pasa de los limitados intereses personales a la preocupación por el bienestar de la comunidad. Los esteticistas evolutivos han visto a menudo el arte, o las habilidades que este implica, como una posibilidad de unir a 
las comunidades, dándoles a estas una ventaja adaptativa. El asombro, y aquello que permite su surgimiento, podría ser adaptativo en este sentido. Todo ello tiene que ver con un asunto más amplio, como lo es la relación entre estética y ética. Si el asombro en la vida cotidiana está íntimamente asociado con la ética, entonces existe entre ellos un vínculo más estrecho de lo que habíamos pensado ${ }^{16}$. Piff y Keltner (2015) aseguran que en una de sus investigaciones encontraron que algunos de los participantes del estudio que dijeron haber vivido más momentos de asombro en sus vidas, y que aquellos que regularmente percibían belleza y asombro en el mundo a su alrededor, eran más amables con los extraños. Las experiencias de asombro podrían hacer que uno se sienta más expansivo y menos impulsado a satisfacer necesidades personales inmediatas.

Piff y Keltner (2015) también han señalado que nuestra cultura se priva de las experiencias de asombro en la medida en que gastamos mucho más tiempo trabajando que compartiendo al aire libre con otras personas. Los jóvenes de hoy se pierden las posibilidades que ofrecen ir a campar y ver un cielo estrellado, algo que hacía la actual gente adulta en su juventud. Kant (2013) decía que "Dos cosas Ilenan la mente de una admiración y asombro siempre nuevas, algo sobre lo cual reflexionamos más a menudo y más constantemente: los cielos estrellados en las alturas y la ley moral interna" (s.p). Piff y Keltner (2015) plantean que al experimentar el asombro mientras miramos los cielos estrellados, estamos más inclinados a seguir la ley moral, o al menos a ayudar a otros. Los mismos autores observaron un descenso en la asistencia a eventos artísticos junto con una disminución en la financiación de programas de arte en los colegios, y concluyen que la "privación de experiencias de asombro ha influido en un cambio social amplio en los últimos cincuenta años", en la medida en que "la gente se ha vuelto más individualista, más centrada en sí misma y menos conectada con los demás" (Piff \& Keltner, 2015, p. 10). 
Es difícil medir lo anterior, y uno debería tener cuidado al momento de definir los conceptos clave. La consecuencia, sin embargo, si es verdadera, es que la estética, incluyendo la estética cotidiana, puede ser más importante — de lo que muchos creen actualmente- para nuestra supervivencia cultural. Piff y Keltner (2015) concluyen que, para invertir la tendencia, la gente experimenta más asombro en la cotidianidad, buscando activamente aquello "que le eriza la piel, bien sea mirando los árboles, los cielos nocturnos, los patrones de viento, del agua o la generosidad diaria de los otros... (s.p) ${ }^{\prime \prime 17}$. Promover esto sería un objetivo importante para la estética cotidiana, pero solo desde una definición expansiva.

Poner el énfasis en lo ordinario por encima de lo extraordinario y lo común sobre lo asombroso en la estética cotidiana es como juzgar el arte por sus ejemplos más mundanos y no por sus obras maestras. El equivalente a una obra maestra en la estética cotidiana es aquello a lo que Dewey (2005) domina como "una experiencia", lo cual está asociado a lo que podemos llamar asombro ${ }^{18}$. La estética cotidiana puede tener (¡debería tener!) una dimensión normativa en el sentido de proporcionar niveles altos, cosas a las que pueda aspirar. Puede aspirar a incrementar los placeres y disminuir las penas de nuestras vidas, no sólo cuantitativamente, como lo sugiere el énfasis de Melchionne (2014) con la regulación hedónica según Bentham (1988), sino también maximizando los momentos de asombro. Dichos momentos, sin embargo, no son actividades o prácticas. Esto es cierto, aunque uno puede cultivar la obtención de estas experiencias de manera similar a la práctica budista de la atención plena.

\footnotetext{
${ }^{17}$ Aunque no lo discutiremos aquí, está abierta la inquietud sobre si que la piel se erice es necesario o suficiente en las experiencias de asombro. ${ }^{18}$ Para conocer mi opinión sobre el concepto de "una experiencia" según Dewey, ver Leddy (2012a, pp. 57-58).
} 


\section{La "hora de la basura"}

El punto del desacuerdo entre Melchionne (2013) y yo puede subrayarse al observar su elaboración acerca del hecho de sacar la basura. Melchionne (2013) afirma que se trata de "una actividad cotidiana casi para todo el mundo, pero no es típicamente estética. Sería raro embellecerla ceremoniosamente". Admite que es posible concebirla como tal, pero que "lo que importa no es la posibilidad lógica de una cualidad sino su tipicidad" (2d).

De acuerdo, sacar la basura no es considerado una actividad típicamente estética o algo que pueda tener una cualidad estética positiva. Pero, ¿debería esto excluirse de la estética cotidiana? Una de mis tiras cómicas favoritas, Rose is Rose ${ }^{19}$, pone regularmente al esposo "Jimbo", uno de los protagonistas, a experimentar algo que él Ilama su "hora de la basura". Cuando saca la basura, este personaje contempla las estrellas, el universo y la vida. Para él esta tarea tiene un profundo carácter estético; quizás sea sublime. Por otra parte, y debido a esto, existe una cierta cualidad ceremonial al sacar su basura. Coincido con Melchionne (2013) en que resulta extraño embellecer esta práctica y ponerla al nivel de verdaderas ceremonias como la lectura de ciertos versos, encender velas, o la ceremonia japonesa del té. No obstante, no es tan raro, mientras se saca la basura, parar y mirar al cielo, o pensar en el rol de cada uno en el universo. Tampoco es solo el pensamiento sobre el universo lo que proporciona la experiencia del asombro. El asombro de la "hora de la basura" surge de la experiencia en su conjunto.

Por supuesto, parte de la experiencia es la ironía que implica algo normalmente considerado una tarea común. Después de todo, Rose is Rose es una historieta y, de algún modo, es chistoso que Jimbo tenga horas de la basura. Con todo, el humor no excluye lo estético. Además, no hay claridad en que asumir sacar 
la basura desde una perspectiva estética en conexión con el asombro sea algo malo. En una dirección similar, Thich Nhat Hanh (1987), siguiendo la tradición budista, nos sugiere lavar los platos mediante lo que él denomina atención plena: "Al lavar los platos, solo se debería estar lavando los platos, lo que significa que uno debería estar completamente consciente del hecho de que uno está lavando los platos" (4). Esto podría ser malinterpretado al entenderse como si sólo debiéramos concentrarnos en la verdad de la proposición "Estoy lavando los platos". Más bien, lo que significa es que uno debería ser consciente de cada una de las acciones que supone lavar los platos cuando hace dicha actividad. Quizá, uno debería también ser consciente de los platos, en la medida en que son parte de la experiencia total y de sus propiedades estéticas, aunque Hanh (1987) no menciona nada al respecto ${ }^{20}$.

Del mismo modo, al describir el consumo consciente de té, Hanh (1987) escribe: "Bebe tu té despacio y con reverencia, como si fuera el eje en el que gira toda la Tierra lenta y uniformemente sin precipitarse hacia el futuro. Vive el momento presente. La vida solo ocurre en el momento actual" (42). Desde mi punto de vista, esta manera de tomar el té le permitiría a uno concentrarse en las cualidades estéticas del té, del instante como experiencia y, también, en nuestras propias acciones ${ }^{21}$. La exigencia estética, como en el budismo, es percibir el ahora para vivir. Como expresa Hanh (1987): “Tomar una taza de té se convierte en una experiencia directa y maravillosa, en la cual la diferencia entre el sujeto y el objeto ya no existe" (42). Si eso sucede, la experiencia ya no es común u ordinaria y, sin embargo, lo ordinario se eleva e intensifica.

Melchionne (2013) desarrolla el asunto de sacar la basura planteando que una práctica cotidiana no se convierte en estética por alguna "transfiguración contra-intuitiva o una ocurrencia de reinvención creativa" (4), parecido a lo

\footnotetext{
${ }^{20}$ Yuriko Saito me ha señalado que, de manera similar, la práctica zen no discrimina en contra de actividades tan simples como limpiar los pisos o el aseo personal. El sacerdote zen japonés del siglo XII, Dogen (2007), dejó algunos escritos relacionados con estas prácticas..

${ }^{21}$ Richard Shusterman, igualmente, interpreta el budismo desde esta perspectiva estética. Ver Shusterman (2012, pp. 288-314).
} 
que pasó cuando Duchamp creó El urinario. De acuerdo, pero no se necesita algo tan vanguardista o contra-intuitivo para tener lo que hemos denominado la "hora de la basura". La hora de la basura no consiste en convertir algo ordinario en una obra de arte.

Dewey (2005) nos insta no solo a notar las propiedades estéticas de una fuerte tormenta o de una comida maravillosa en un restaurante, sino también a ver tales propiedades en el fuego de la chimenea y en la satisfacción estética que le produce a un mecánico su trabajo bien hecho. En mi libro (Leddy, 2012a), enfatizo el valor de adoptar una actitud estética hacia cosas tan ordinarias como las sombras de los árboles en las aceras. Irvin (2008), de manera similar, ha subrayado tal actitud cuando, por ejemplo, observa a su gato o, incluso, las forma en que ella se sienta y respira. Estas últimas cosas no nos sacan de la corriente de la vida cotidiana, sino que implica asumir una actitud diferente hacia dicha corriente ${ }^{22}$.

\section{Conclusión}

En conclusión, he problematizado los planteamientos de Melchionne (2013, 2014) y Naukkarinen (2013) que restringen la estética cotidiana a "los aspectos de nuestra vida marcados por rutinas o pautas cotidianas ampliamente compartidos a los cuales tendemos a asignarles un carácter estético" (Melchionne, 2013, p. 3). Aunque valoro que hayan Ilamado nuestra atención sobre tales asuntos, sostengo que las cosas no tan ampliamente compartidas y no tan cotidianas, habituales o comunes, también pueden tenerse en cuenta en la estética cotidiana. Es necesaria una definición expansiva de la estética cotidiana, pues la regulación hedónica no es suficiente para el bienestar subjetivo; también requerimos instantes de asombro. En parte, esto puede proporcionarse a través de las estéticas del arte y la naturaleza. Sin embargo, si tomamos el camino

\footnotetext{
${ }^{22}$ De nuevo, Moore (1996) va en otra dirección intentando reincorporar los asuntos del festival, la magia y la mitología en la estética cotidiana.
} 
de Jimbo o Hanh, estos momentos de asombro pueden emerger por fuera de las experiencias cotidianas de la vida diaria, adoptando una perspectiva correcta y teniendo una adecuada educación. No entendamos la estética cotidiana como una mera bolsa adicional para echar lo que no es arte ni naturaleza. Pero tampoco seamos demasiado restrictivos, ni estemos demasiado atados al mérito de eso que hace fácil y cómodo reconocer el valor de lo extraño, interesante, fascinante y asombroso de la vida cotidiana. La búsqueda de esto puede hacer que la vida, como afirmara Nietzsche, valiera la pena vivirse. Y si se define la estética cotidiana de una manera restrictiva, estamos restringiendo la vida misma ${ }^{23}$.

\section{Referencias}

Bentham, J. (1988). The Principles of Morals and Legislation. Amherst: Prometheus Books.

Berleant, A. (1964). The Sensuous and the Sensual in Aesthetics. Journal of Aesthetics and Art Criticism, 23(2), 185-192.

Burke, E. (1990). A Philosophical Enquiry into the Origin of our Ideas of the Sublime and Beautiful. New York: Oxford University Press.

Carlson, A. (1979). Appreciation and the Natural Environment. Journal of Aesthetics and Criticism, 37(3), 267-275.

Davies, S. (2015). The Artful Species. Oxford: Oxford University Press.

Dewey, J. (2005). Arts as Experience. New York: Perigree.

Dickie, G. (1971). Aesthetics: An introduction. Indianapolis: Pegasus, BobbsMerrill.

\footnotetext{
${ }^{23}$ Quiero agradecer a Kevin Melchionne, Ossi Naukkarinen y Yuriko Saito por brindarme comentarios de mucha ayuda para las versiones iniciales de estas ideas.
} 
Dissanayake, E. (1992). Homo Aestheticus: Where Art Comes from and Why. New York: Free Press.

Dogen, E. (2007). Shobogenzo: The True Dharma-Eye Treasury. Volume I (Taisho Volume 82, Number 2582). (G.W. Nishijima \& C. Cross, trds.). Bukkyo Dendo Kyokai and Nomata Center for Buddhist Translation. Recuperado de http://www.bdkamerica.org/digital/dBET_T2582_Shobogenzo1_2009.pdf

Dutton, D. (2010). The Art Instinct. New York: Bloomsbury Press.

Fitzgerald, F. S. (2011). Winter Dreams. En Th. Fasano (Ed.), Great Short Stories by Great American Writers (pp. 382-399). Claremont: Coyote Canyon Press.

Haapala, A. (2005). On the aesthetics of everyday: familiarity, strangeness and the meaning of place. En A. Light \& J.M. Smith (Eds.), The aesthetics of everyday life (pp.39-55). New York: Columbia University Press.

Hanh, T. N. (1987). The Miracle of Mindfulness (M. Ho, tr.). Boston: Beacon Press.

Irvin, S. (2008). The Pervasiveness of the Aesthetic in Ordinary Experience. British Journal of Aesthetics, 48, 29-44.

Kant, I. (2013). The Critique of Practical Reason (T. Kingsmill Abbot, tr.). Proyecto Gutenberg. Recuperado de http://www.gutenberg.org/ebooks/5683

Keltner, D. \& Haidt, J. (2003). Approaching Awe, A Moral, Spiritual, And Aesthetic Emotion. Cognition and Emotion, 17, 297-314.

Leddy, T. (2012a). The extraordinary in the ordinary. Peterborough: Broadview Press. 
Leddy, T. (2012b). Defending everyday aesthetics and the concept of pretty. Contemporary Aesthetics, 10. Recuperado de http://www. contempaesthetics.org/newvolume/pages/article.php?articleID=654\&searc hstr=leddy

Leddy, T. (2014). Everyday Aesthetics and Happiness. En L. Yuedi \& C. Carter (Eds.), Aesthetics of Everyday Life: West and East (pp. 26-47). New Castle upon Tyne: Cambridge Scholars Publishing.

Lintott, S. (2012). The Sublimity of Getating and Giving Birth: Toward a Feminist Conception of the Sublime. En S. Lintott \& M. Sander-Staudt (Eds.), Philosophical Inquiries into Pregnancy, Childbirth, and Mothering: Maternal Subjects (pp. 237-250). London: Routledge

Melchionne, K. (2013). The definition of everyday aesthetics. Contemporary Aesthetics, 11. Recuperado de http://www.contempaesthetics.org/ newvolume/pages/article.php?articlelD=663 \&searchstr=melchionne

Melchionne, K. (2014). The point of everyday aesthetics. Contemporary Aesthetics, 12. Recuperado de http://www.contempaesthetics.org/ newvolume/pages/article.php?articleID=700\&searchstr=melchionne

Mikulak, A. (2015). All about Awe: Science Explores How Life's Small Marvels Elevate Cognition and Emotion. Observer, 28, 4. Recuperado de http://www.psychologicalscience.org/issue/april-15Moore, T. (1996). The ReEnchantment of Everyday Life. New York: Harper Collins.

Naukkarinen, O. (2013). What is "Everyday" in Everyday Aesthetics? Contemporary Aesthetics, 11. Recuperado de http://www.contempaesthetics. org/newvolume/pages/article.php?articleID $=675$

Piff, P. \& Keltner, D. (2015, May 24). Why Do We Experience Awe? New York Times (Sunday Review), p. 10. 
Plutchik, R. (s.f). Contrasting and Categorization of Emotions. En Wikipedia. Recuperado de https://en.wikipedia.org/wiki/Contrasting_and_categorization_ of_emotions\#Plutchik.27s_wheel_of_emotionsSaito, Y. (2010). Everyday Aesthetics. Oxford: Oxford University Press.

Shusterman, R. (2007). Asian Ars Erotica and the Question of Sexual Aesthetics. The Journal of Aesthetics and Art Criticism, 65, 55-68.

Shusterman, R. (2012). Thinking Through the Body: Essays on Somaesthetics. Cambridge: Cambridge University Press. Wilde, O. The Decay of Lying. Recuperado de http://virgil.org/dswo/courses/novel/wilde-lying.pdf

Como citar: Leddy. T. y Pérez-Henao, H. (2020). La experiencia del asombro: una aproximación expansiva a la estética cotidiana. Revista KEPES, 17(22), 397-425. https://doi.org/10.17151/kepes.2020.17.22.15 